Научная статья

УДК: 531.36

DOI: $10.18101 / 2304-5728-2020-3-42-48$

\title{
ОБ УСТОЙЧИВОСТИ ОДНОГО ВИДА ПЕРМАНЕНТНЫХ ВРАЩЕНИЙ МЕХАНИЧЕСКОЙ СИСТЕМЫ С ЧАСТНЫМ ИНТЕГРАЛОМ ГЕССА
}

\section{(C) Новиков Михаил Алексеевич}

доктор физико-математических наук, старший научный сотрудник, Институт динамики систем и теории управления имени В. М. Матросова СО РАН

Россия, 664033, г. Иркутск, ул. Лермонтова, 134 nma@icc.ru

Благодарности. Работа выполнена при поддержке РФФИ (проект N 19-08-00746).

Аннотация. В заметке рассматривается механическая автономная консервативная система с частным интегралом Гесса в известной задаче о вращении твердого тела вокруг неподвижной точки. Указанная система описывается шестью обыкновенными дифференциальными уравнениями. Проведено исследование устойчивости по Ляпунову четырех видов стационарных движений, для которых отличны от нуля все компоненты углов Пуассона. Предложенные стационарные движения являются перманентными вращениями. Исследование опирается на первый метод Ляпунова. С этой целью для дифференциальных уравнений возмущенного движения по матрице правой части в линейном приближении составляется характеристическое уравнение. Получение алгебраических выражений, их упрощение и факторизация осуществляется системой аналитических вычислений на современных персональных компьютерах. Для всех перманентных вращений установлены одинаковые характеристические уравнения. Анализ коэффициентов полученных уравнений позволил установить, кроме нулевых и чисто мнимых корней, наличие двух вещественных решений, отличных от нуля. В консервативных системах один из них будет положительным. Проведенные вычисления показали неустойчивость всех исследуемых перманентных вращений.

Ключевые слова: механическая автономная консервативная система; дифференциальные уравнения движения; стационарное движение; перманентное вращение; общий интеграл; частный интеграл; возмущенное движение; характеристическое уравнение; корни алгебраического уравнения; необходимые условия устойчивости; неустойчивость движения.

\section{Для цитирования}

Новиков М. А. Об устойчивости одного вида перманентных вращений механической системы с частным интегралом Гесса // Вестник Бурятского государственного университета. Математика, информатика. 2020. № 3. С. 42-48. 
M. А. Новиков. Об устойчивости одного вида перманентных вращений механической системы с частным интегралом Гесса

\section{Введение}

В задаче о движении твердого тела вокруг неподвижной точки $[1 ; 2]$ кроме трех известных общих интегралов приведены случаи существования некоторых частных интегралов [2]. Механическая автономная консервативная система с частным интегралом Гесса описывается дифференциальными уравнениями:

$$
\left\{\begin{array}{cc}
A \dot{p}=(B-C) q r+z_{0} \gamma_{2}, & \dot{\gamma}_{1}=r \gamma_{2}-q \gamma_{3}, \\
B \dot{q}=(C-A) r p-z_{0} \gamma_{1}+x_{0} \gamma_{3}, & \dot{\gamma}_{2}=p \gamma_{3}-r \gamma_{1}, \\
C \dot{r}=(A-B) p q-x_{0} \gamma_{2}, & \dot{\gamma}_{3}=q \gamma_{1}-p \gamma_{2},
\end{array}\right.
$$

где $x_{0} \neq 0 \neq z_{0} ; A, B, C-$ моменты инерции твердого тела относительно главных осей $O x, O y, O z ; p, q, r$ - проекции мгновенной угловой скорости на подвижные, связанные с телом оси; $x_{0}, z_{0}$ - координаты центра масс в подвижных осях; $\gamma_{1}, \gamma_{2}, \gamma_{3}$ - проекции ортов подвижных осей на неподвижную вертикальную ось $O Z$ (углы Пуассона).

Для системы (1) известны три общих интеграла [2]:

$$
\begin{aligned}
& V_{0}=A p^{2}+B q^{2}+C r^{2}+2\left(x_{0} \gamma_{1}+z_{0} \gamma_{3}\right)=\text { const }, \\
& V_{1}=A p \gamma_{1}+B q \gamma_{2}+C r \gamma_{3}=\text { const }, \\
& V_{2}=\gamma_{1}^{2}+\gamma_{2}^{2}+\gamma_{3}^{2}=1 .
\end{aligned}
$$

В системе (1) при выполнении равенства Аппельрота [2]:

$$
A C\left(x_{0}^{2}+z_{0}^{2}\right)=B\left(A x_{0}^{2}+C z_{0}^{2}\right)
$$

возможно существование частного линейного интеграла Гесса, записанного в аналитическом виде

$$
V_{3}=A x_{0} p+C z_{0} r=0 .
$$

При изучении основных динамических свойств механических систем важное значение занимает исследование устойчивости стационарных движений.

\section{1 Постановка задачи}

В статье [3] приведены некоторые виды стационарных движений системы (1). Не рассматривая в отдельности знаки параметризованной величины $\gamma_{10}$, часть исследуемых стационарных движений можно отнести к одной группе с отличными от нуля значениями $\gamma_{20}$ :

1. $p_{0}=P ; q_{0}=Q ; r_{0}=-R ; \gamma_{20}=G_{2} ; \gamma_{30}=G_{3}$,

2. $p_{0}=P ; q_{0}=-Q ; r_{0}=-R ; \gamma_{20}=-G_{2} ; \gamma_{30}=G_{3}$,

3. $p_{0}=-P ; q_{0}=Q ; r_{0}=R ; \gamma_{20}=-G_{2} ; \gamma_{30}=G_{3}$,

4. $p_{0}=-P ; q_{0}=-Q ; r_{0}=-R ; \gamma_{20}=G_{2} ; \gamma_{30}=G_{3}$, 
где

$P=\frac{1}{A(A-C) x_{0}} \sqrt{A(A-C)\left(A x_{0}^{2}+C z_{0}^{2}\right) x_{0} \gamma_{10}}$,

$Q=\frac{1}{C z_{0}} \sqrt{\frac{\left(A x_{0}^{2}+C z_{0}^{2}\right)\left[C^{2} z_{0}^{2}-\left(A^{2} x_{0}^{2}+C^{2} z_{0}^{2}\right) \gamma_{10}^{2}\right]}{A(A-C) x_{0} \gamma_{10}}}$,

$R=\frac{1}{C(A-C) z_{0}} \sqrt{A\left(A x_{0}^{2}+C z_{0}^{2}\right)(A-C) x_{0} \gamma_{10}}$,

$G_{2}=\frac{\sqrt{C^{2} z_{0}^{2}-\left(A^{2} x_{0}^{2}+C^{2} z_{0}^{2}\right) \gamma_{10}^{2}}}{C z_{0}}, G_{3}=\frac{-A x_{0} \gamma_{10}}{C z_{0}}$.

Действительные перманентные вращения (1.1)-(1.4) при $\gamma_{20} \neq 0$ могут существовать только для положительных (во всяком случае неотрицательных) покоренных выражений $P, Q, R, G_{2}, \quad$ когда выполняется неравенства:

$$
(A-C) x_{0} \gamma_{10}>0, \quad \gamma_{10}^{2}<\frac{C^{2} z_{0}^{2}}{A^{2} x_{0}^{2}+C^{2} z_{0}^{2}} .
$$

Предельные значения $\gamma_{10}= \pm \frac{C z_{0}}{\sqrt{A^{2} x_{0}^{2}+C^{2} z_{0}^{2}}}$ здесь не рассматриваются, их можно отнести к другому виду стационарных движений при $q_{0}=0, \gamma_{20}=0$.

Ставится цель исследования устойчивости по Ляпунову представленных перманентных вращений (1.1)-(1.4) при условиях (1.5).

\section{2 Необходимые условия устойчивости}

Следуя [4-7], прежде всего нужно установить ограничения на динамические и статические параметры системы (1), для которых выполняются необходимые условия устойчивости. Во всяком случае при необходимости это может оказаться востребованным для сопоставления с достаточными условиями устойчивости. Для этого составим отклонения:

$$
\begin{gathered}
x_{1}=p-p_{0} ; x_{2}=q-q_{0} ; x_{3}=r-r_{0} ; \\
x_{4}=\gamma_{1}-\gamma_{10} ; x_{5}=\gamma_{2}-\gamma_{20} ; x_{5}=\gamma_{3}-\gamma_{30} .
\end{gathered}
$$

$\mathrm{B}$ автономных системах важное значение имеет матрица правой части линеаризованной системы дифференциальных уравнений возмущенного движения. Для перманентного вращения (1.1) она имеет следующее представление: 
M. А. Новиков. Об устойчивости одного вида перманентных вращений механической системы с частным интегралом Гесса

$$
D=\left(\begin{array}{cccccc}
0 & \frac{(B-C) r_{0}}{A} & \frac{(B-C) q_{0}}{A} & 0 & \frac{z_{0}}{A} & 0 \\
\frac{(C-A) r_{0}}{B} & 0 & \frac{(C-A) p_{0}}{B} & \frac{-z_{0}}{B} & 0 & \frac{x_{0}}{B} \\
\frac{(A-B) q_{0}}{C} & \frac{(A-B) p_{0}}{C} & 0 & 0 & \frac{-x_{0}}{C} & 0 \\
0 & -\gamma_{30} & 0 & 0 & r_{0} & -q_{0} \\
\gamma_{30} & 0 & -\gamma_{10} & -r_{0} & 0 & p_{0} \\
0 & \gamma_{10} & 0 & q_{0} & -p_{0} & 0
\end{array}\right) .
$$

При подстановке из равенства Аппельрота величины $B=\frac{A C\left(x_{0}^{2}+z_{0}^{2}\right)}{A x_{0}^{2}+C z_{0}^{2}}$ характеристическое уравнение для матрицы $D$ имеет вид

$$
f(\lambda)=\operatorname{det}(D-\lambda E)=\lambda^{2} f_{1}(\lambda)=0,
$$

так что

$$
\begin{gathered}
f_{1}(\lambda)=\lambda^{4}+a_{2} \lambda^{2}+a_{0} \\
\text { где } a_{2}=\frac{F_{2}}{A C^{2}(A-C) x_{0} \gamma_{10}\left(A x_{0}^{2}+C z_{0}^{2}\right)\left(x_{0}^{2}+z_{0}^{2}\right)} ; \\
\quad a_{0}=\frac{F_{0}}{A^{2} C^{4}\left(x_{0}^{2}+z_{0}^{2}\right) \gamma_{10}^{2}} ; \\
\quad F_{2}=A^{2}\left[C^{2}+4(A-C)^{2} \gamma_{10}^{2}\right] x_{0}^{6}+ \\
\quad+\left[C^{3}(4 A-C)+\left((C-A)^{4}+8 A C(A+C)^{2}\right) \gamma_{10}^{2}\right] x_{0}^{4} z_{0}^{2}+ \\
+C^{2}\left[A(4 A-C)+4(A-C)^{2} \gamma_{10}^{2}\right] x_{0}^{2} z_{0}^{4}+C^{4} z_{0}^{6} ; \\
F_{0}=-\left[C^{2}\left(x_{0}^{2}+z_{0}^{2}\right)+3 x_{0}^{2}(A-C)^{2} \gamma_{10}^{2}\right]\left[C^{2} z_{0}^{2}-\left(A^{2} x_{0}^{2}+C^{2} z_{0}^{2}\right) \gamma_{10}^{2}\right]
\end{gathered}
$$

Здесь для упрощения и контроля вычислений определителя матрицы, подстановок, замены переменных и факторизации символьных выражений применялась система аналитических вычислений "Mathematica" на персональном компьютере.

При анализе корней характеристического уравнения наиболее важным является определение знака свободного члена. Его знаменатель представляет положительное выражение как произведение только положительных величин.

Легко видеть, что в выражении $F_{0}$ первая квадратная скобка положительна, и вторая квадратная скобка для значений $\left|\gamma_{10}\right|$ из условия (1.5) принимает только положительные значения.

Так как $a_{0}<0$, то независимо от знака $F_{2}$ биквадратное уравнение (2.1) имеет два чисто мнимых корня и два вещественных, из которых 
один корень положительный. По соответствующей теореме Ляпунова [4-7] перманентное вращение (1.1) тогда будет неустойчивым.

Ввиду недопустимости выражения $Q$ при $\gamma_{10}=0$ стационарных движений (1.1)-(1.4) будут рассматриваться только значения $\gamma_{10} \neq 0$.

Можно показать, что характеристические уравнения для матриц возмущенного движения перманентных вращений (1.2)-(1.4) получаются точно такими же. Для них также имеет место неустойчивость перманентных вращений.

Окончательно можно сформулировать результат.

Tеорема. Перманентные вращения механической системы (1), допускающей частный интеграл Гесса, для которых все составляющие углов Пуассона отличны от нуля, являются неустойчивыми.

Следует отметить, что в стационарных движениях (1.1)-(1.4) на выбор знаков $x_{0}, z_{0}, \gamma_{10}$ влияют только ограничения (1.5). При этом допускаются различные сочетания знаков $x_{0}, z_{0}$, и расположение центра масс ниже или выше горизонтальной плоскости здесь несущественно.

\section{Заключение}

В статье [8] изучена устойчивость состояния покоя системы (1) при значениях $p_{0}=q_{0}=r_{0}=\gamma_{20}=0$. Устойчивость установлена при расположении центра масс ниже начала координат, в ином случае неустойчивость.

В [9] для перманентных вращений при $\gamma_{20}=0$ показана возможность выполнения необходимых условий устойчивости при определенных соотношениях на моменты инерции $A$ и $C$. Конечно, при этих соотношениях могут выполняться и достаточные условия устойчивости.

Последние виды стационарных движений, существующие при $\gamma_{20} \neq 0$, рассмотрены в настоящей статье, где установлена невозможность устойчивости при любых динамических и статических параметрах системы (1). Притом неустойчивость получена по линейному приближению, когда не выполняются необходимые условия устойчивости.

\section{Литература}

1. Аппель П. Теоретическая механика. М.: ГИФМЛ, 1960. Т. 2. 487 с.

2. Голубев В. В. Лекции по интегрированию уравнений движения тяжелого твердого тела около неподвижной точки. М.: Регулярная и хаотическая динамика, 2002. $287 \mathrm{c}$.

3. Новиков М. А. О стационарных движениях твердого тела при существовании частного интеграла Гесса // Известия РАН. Механика твердого тела. 2018. № 3. С. 28-37.

4. Ляпунов А. М. Общая задача об устойчивости движения // Собрание сочинений. М.-Л.: Изд-во АН СССР, 1956. Т. 2. С. 7-263. 
M. А. Новиков. Об устойчивости одного вида перманентных вращений механической системы с частным интегралом Гесса

5. Четаев Н. Г. Устойчивость движения. Работы по аналитической механике. М.: Изд-во АН СССР, 1962. 535 с.

6. Каменков Г. В. Устойчивость движения, колебания, аэродинамика. М.: Наука, 1971. Т. 1.255 с.

7. Каменков Г. В. Устойчивость и колебания нелинейных систем. М.: Наука, 1972. Т. 2. 213 c.

8. Новиков М. А. Об устойчивости стационарных движений транспортных систем при существовании частного интеграла // Современные технологии. Системный анализ. Моделирование. 2019. № 4 (64). С. 57-64.

9. Новиков М. А. Об устойчивости стационарного движения механической консервативной системы // Вестник Бурятского государственного университета. Математика, информатика. 2018. № 3. С. 22-39.

\title{
ON THE STABILITY OF ONE TYPE OF PERMANENT ROTATIONS OF A MECHANICAL SYSTEM WITH A HESSIAN PATICULAR INTEGRAL
}

\author{
Mikhail A. Novikov \\ Dr. Sci. (Phys. and Math.), Senior Researcher, \\ Matrosov Institute for System Dynamics and Control Theory SB RAS \\ 134 Lermontova St., Irkutsk 664033, Russia \\ nma@icc.ru
}

Abstract. The paper considers a mechanical autonomous conservative system with a Hessian particular integral in the well-known problem of the rotation of a rigid body around a fixed point. This system is described by six ordinary differential equations. We have carried out a study of the Lyapunov stability of four types of stationary motions for which all components of the Poisson angles are nonzero. The proposed stationary movements are permanent rotations. The study is based on the first Lyapunov method. For this purpose, we derive a characteristic equation for the differential equations of disturbed motion on the matrix of the right-hand side in the linear approximation. Obtaining algebraic expressions, their simplification and factorization are carried out by a system of analytical calculations on modern personal computers. The same characteristic equations are established for all permanent rotations. The analysis of the coefficients of the obtained equations made it possible to establish, in addition to zero and purely imaginary roots, the presence of two real solutions other than zero. In conservative systems, one of them will be positive. The calculations performed showed the instability of all the studied permanent rotations.

Keywords: mechanical autonomous conservative system; differential equations of motion; stationary motion; permanent rotation; general integral; particular integral; perturbed motion; characteristic equation; roots of an algebraic equation; necessary conditions of stability; instability of motion.

\section{References}

1. Appell P. Teoreticheskaya mekhanica [Theoretical Mechanics]. Moscow: GIFML Publ., 1960. Vol. 2. 487 p.

2. Golubev V. V. Lektsii po integrirovaniyu uravnenii dvizheniya tyazhelogo tverdogo tela okolo nepodvizhnoi tochki [Lectures on Integration of Equations of Motion for a Heavy Rigid Body about a Fixed Point]. Moscow: Regulyarnaya i khaoticheskaya dinamika Publ., 2002. 287 p. 
3. Novikov M. A. O statsionarnykh dvizheniyakh tverdogo tela pri sushchestvovanii chastnogo integrala Gessa [On Steady-State Motions of a Rigid Body in the Case of the Existence of Hessian Particular Integral]. Mechanics of Solids. 2018. No. 3. Pp. 28-37.

4. Lyapunov A. M. Obshchaya zadacha ob ustoichivosti dvizheniya. Sobraniye sozhineniy [The General Problem of the Stability of Motion. Collected Works]. Moscow-Leningrad: USSR AS Publ., 1956. Vol. 2. Pp. 7-263.

5. Chetayev N. G. Ustoychivost Ustoichivost' dvizheniya. Raboty po analiticheskoi mekhanike [Stability of Motion. Works on Analytical Mechanics]. Moscow: USSR AS Publ., 1962. 535 p.

6. Kamenkov G. V. Ustoichivost dvizheniya, kolebaniya, aerodinamika [Stability of Motion, Vibrations, Aerodynamics]. Moscow: Nauka Publ., 1971. Vol. 1. 255 p.

7. Kamenkov G. V. Ustoichivost $i$ kolebaniya nelineinykh sistem [Stability and Vibrations of Nonlinear systems]. Moscow: Nauka Publ., 1972. Vol. 2. 213 p.

8. Novikov M. A. Ob ustoichivosti statsionarnykh dvizhenii transportnykh sistem pri sushchestvovanii chastnogo integrala [On Stability of Steady-State Motions of Transport Systems in the Case of Existence of a Particular Integrals]. Sovremennye tekhnologii. Sistemnyi analiz. Modelirovanie. 2019. No. 4 (64). Pp. 57-64.

9. Novikov M. A. Ob ustoichivosti statsionarnogo dvizheniya mekhanicheskoi konservativnoi sistemy [On the Stability of Stationary Motions of a Mechanical Conservative System]. Vestnik Buryatskogo gosudarstvennogo universiteta. Matematika i informatika. 2018. No. 3. Pp. 22-39.

Статья поступила в редакцию 18.11.2020; одобрена после рецензирования 30.11.2020; принята к публикации 10.12.2020. 